\title{
Activity of the Leonid meteor shower on 2009 November 17
}

\author{
P. Koten ${ }^{1}$, J. Borovička ${ }^{1}$, and G. I. Kokhirova ${ }^{2}$ \\ 1 Astronomical Institute of the Academy of Sciences, Ondřejov Observatory, Fričova 298, 25165 Ondřejov, Czech Republic \\ e-mail: koten@asu.cas.cz \\ 2 Institute of Astrophysics, Tajik Academy of Sciences, 734042 Dushanbe, Tajikistan
}

Received 26 November 2010 / Accepted 21 January 2011

\section{ABSTRACT}

\begin{abstract}
Context. The double-station video experiment was established in Tajikistan to confirm the prediction of the Leonid meteor shower in 2009 activity connected with the material that was ejected from the parent comet in 1466 and 1533 .

Aims. Modelling of the meteor shower activity has progressed significant in time-precision in recent years. Enhanced activity of the meteor shower provided us with another opportunity to compare observed results and model predictions. We carried out the doublestation experiment to obtain meteor counts, masses, atmospheric trajectories, and heliocentric orbits.

Methods. The Leonid meteor shower was observed for the whole night of the predicted maximum. For the double-station meteors the geocentric and heliocentric orbital parameters were calculated. Its activity curve was constructed. The mass of the meteoroids was determined on the bases of the meteor light curves, then the distribution of the masses was studied, and finally the flux of the meteoroids was calculated. The results were compared with other Leonid stream filaments, which were observed within the past decade.

Results. The enhanced meteor activity indeed occurred within the predicted time. The masses were determined for 99 Leonid meteors. The distribution shows that this old stream does not produce very bright meteors as the streams observed in 1998 to 2002 did. The flux of the meteoroids reaches a maximum value of $0.02 \pm 0.004$ meteoroids per $\mathrm{km}^{2}$ per hour. This value was significantly lower than values observed in previous years. On the other hand, the peak was broader, what is consistent with a scattered, i.e. older filament encounter.
\end{abstract}

Key words. meteorites, meteors, meteoroids - comets: individual: 55/P/Tempel-Tuttle

\section{Introduction}

The Leonid meteor shower is one of the best known meteor showers, especially because of its high activity, which occurs when the parent comet of the shower reaches its perihelion. Most recently, the comet 55P/Tempel-Tuttle passed through the perihelion on 1998 February 28. Spectacular meteor storms were observed in the years 1998 to 2002 . At the same time, the methods for predicting the precise time and activity of the meteor shower have improved significantly. Already, Kondrateva \& Reznikov (1985) predicted maximum of the Leonid activity in 1999 with a precision of 0.01 day. Their method was improved by McNaught \& Asher (1999), who successfully predicted the same peak, which occurred because of an encounter with material ejected from the parent comet around its 1899 perihelion passage.

After 2002 the activity of the Leonid meteor shower decreased. Nevertheless another event of enhanced activity was predicted for 2006 November 19 (e.g. Lyytinen \& Van Flandern 2000; Vaubaillon \& Colas 2005). This activity was really observed with the peak occurring within $10 \mathrm{~min}$ of the predicted time (Jenniskens et al. 2008; Koten et al. 2008).

Even 2006 event did not mean the Leonid period is over. When Vauballion et al. (2005) applied their model to the older particles ejected from the parent comet, they found another enhancement of the stream density in the vicinity of the Earth for the evening hours of 2009 November 17. At this time our planet could encounter particles released from comet 55P/TempelTuttle in 1466 and 1533. As the recent storms were caused by significantly younger particles, this event provides us with the opportunity to probe properties of the older cometary material. The peaks of activity were predicted to occur at 21:44 and 22:02 UT, respectively. Also Maslov (2007) in his summary paper on the Leonid activity covering period between 2001 and 2100 expected a considerable outburst from the 1466 and 1533 trails between 21 and 22 UT on November 17. The annual maximum should occur according to this paper around 9 UT on the same day.

In this paper we report the observation of this event, as well as the comparison of the experimental results with theoretical predictions.

\section{Observations and instrumentation}

Time of the maximum activity was unfavourable for Central Europe. The Leonid radiant was still below the horizon at the time of the predicted activity. An observer located in this region would be able to see the descending branch of the activity curve at best. Moreover, the weather is usually poor at this period of the year in Central Europe. Therefore we were forced to carry out the double-station video experiment at least several time zones to the east. From the geometrical point of view, Central Asia was the best choice. Thanks to long cooperation with Tajik colleagues operating a photographic fireball network in Tajikistan, we were able to establish our experiment in this country.

Two stations of the Tajik fireball network were selected as the base of the double station video experiment. The northern camera was located at the Gissar astronomical observatory $(\lambda=$ $\left.68^{\circ} 40^{\prime} 53.0^{\prime \prime} \mathrm{E}, \phi=38^{\circ} 29^{\prime} 23.3^{\prime \prime} \mathrm{N}\right)$. The southern camera was 
operated from the Kurgan Tube site $\left(\lambda=68^{\circ} 46^{\prime} 18.3^{\prime \prime} \mathrm{E}, \phi=\right.$ $\left.37^{\circ} 51^{\prime} 12.5^{\prime \prime} \mathrm{N}\right)$. This configuration resulted in the azimuth of the southern station to be $356^{\circ}$ (azimuth of the south $=0^{\circ}$ ), and the distance between stations was $111 \mathrm{~km}$.

The observations were carried out during two consecutive nights. The first night was supposed to be instrument test. The night with the predicted maximum followed the next day. During this night the observation conditions were very good, since the sky was clear and only some haze was visible close to the horizon. The Moon was just one day old, so it did not disturb observations.

Each station was equipped with the instrumentation, which consists of the external lens heating, objective lens Arsat $1.4 / 50 \mathrm{~mm}$, image intensifier Mullard XX1332, and either the analogue S-VHS videocamera Panasonic (Gissar) or the digital mini DV camera JVC (Kurgan Tube). A second analogue camera located at Kurgan Tube station was moreover equipped with the spectral grating with 600 grooves per $\mathrm{mm}$ to record the spectra of brighter meteors. All the data were directly recorded by the camera on the video cassettes. This simple method was selected to minimize the electricity requirements of whole experiment.

Later the tapes were searched for meteors using the meteor recognition software MetRec (Molau 1999). All the meteors identified as the Leonids were digitalized and measured using our semi-automatic software MetPho (Koten 2002). On the double station meteors, we applied standard atmospheric trajectory and heliocentric orbit calculation procedures. In the case of single station meteors, we checked that they are members of the Leonid shower and estimated the atmospheric trajectory. If a backward prolonged meteor path passed the Leonid radiant by less than three degrees, we considered them to be Leonid members. Experience from the previous observational campaigns confirms that such single station meteor data are useful for investigating the meteor activity and the mass distribution index, but the higher errors make them unsuitable for studies oriented toward the atmospheric trajectories and heliocentric orbits.

\section{Results}

Due to technical problems, the first night (from 16th to 17th of November) was only covered from the Gissar station. The problems were resolved, and during the main night of the meteor shower activity we were able to carry out a full-time doublestation observation. Within six hours of the observation, about 350 meteors were recorded by at least one of the cameras. From this number 160 of them were later identified as the meteors belonging to the Leonid meteor shower. For 60 of that recorded from both stations, we could calculate atmospheric trajectories and heliocentric orbits with good precision. Another 60 meteors were only single-station cases with approximated orbits. For another 25 meteors we only recorded spectra but no direct image. The rest of the suspected Leonid meteors suffered from the poor geometry and was not useful for additional analyses; moreover, we obtained a dozen good Leonid spectra.

\subsection{Mass distribution and population index}

First, we calculated the mass distribution index. This quantity describes the distribution of meteoroid masses in the shower. The lower the value, the more massive meteoroids in the stream. We also need this value for calculating the flux of the meteoroids. For this calculation, we can use both single and double station meteors. The mass distribution index can be calculated from the

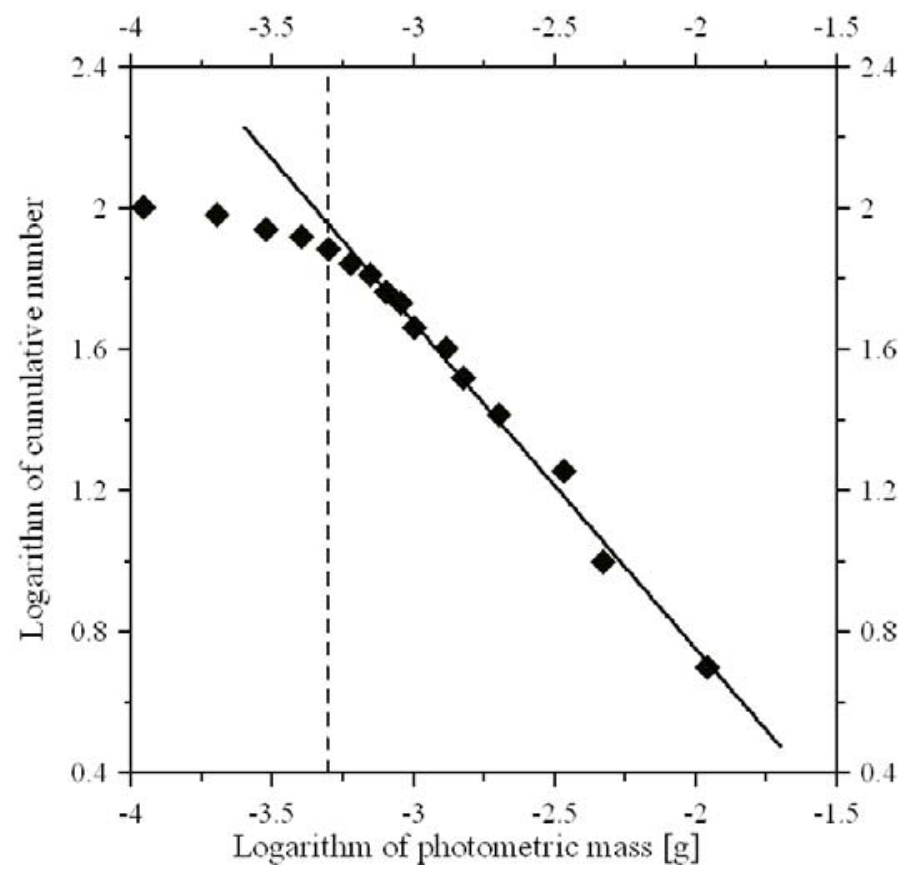

Fig. 1. Mass distribution of the Leonid meteors observed in the night from 17 to 18 November 2009. Solid line represents the linear fit applied on data on the right side of the vertical dashed line. Meteors on its left side, i.e. lower masses, were not taken into account, because their counts are incomplete.

Table 1. Comparison of the mass distribution indices for different Leonid meteor shower filaments with the age of the shower expressed as the year the parent comet perihelion passage during which the material was ejected.

\begin{tabular}{llll}
\hline \hline $\begin{array}{l}\text { Year of } \\
\text { observation }\end{array}$ & $\begin{array}{l}\text { Ejection } \\
\text { from comet }\end{array}$ & $s$ & $\begin{array}{l}\text { Source } \\
\text { of data }\end{array}$ \\
\hline 1999 & 1899 & 1.75 & Brown et al. (2002a) \\
2000 & 1866 & 1.7 & Brown et al. (2002b) \\
2001 & 1767 & 1.75 & Koten (unpublished) \\
2006 & 1932 & 1.9 & Koten et al. (2008) \\
2009 & 1466 & 1.9 & this work \\
\hline
\end{tabular}

slope of the linear part of the plot between the cumulative number of the meteors and their photometric mass (Fig. 1). We could not use the whole range of masses because this curve is not linear in the region of fainter meteors (lower masses). It is simply caused by not being able to detect all the fainter meteors. The photometric mass of each meteoroid was calculated according to the paper of Ceplecha (1988).

Altogether, we could use 99 Leonid meteors with complete light curves for this calculation. For other meteors we could not compute the photometric mass because of incomplete light curves. The 99 meteors were binned in several intervals according to their photometric masses. The slope of the linear fit is $1-s$, where $s$ is the mass distribution index. From Fig. 1 we receive value -0.9 , which results in the index $s=1.9$. This value is similar to the values derived for other Leonid observations within the last decade (Table 1). We can say that the 2009 Leonid meteor shower was slightly richer in fainter meteors than were the earlier showers noted in the table.

From the mass distribution index we can easily determine the population index $r$ using equation

$s=1+2.5 \log r$ 


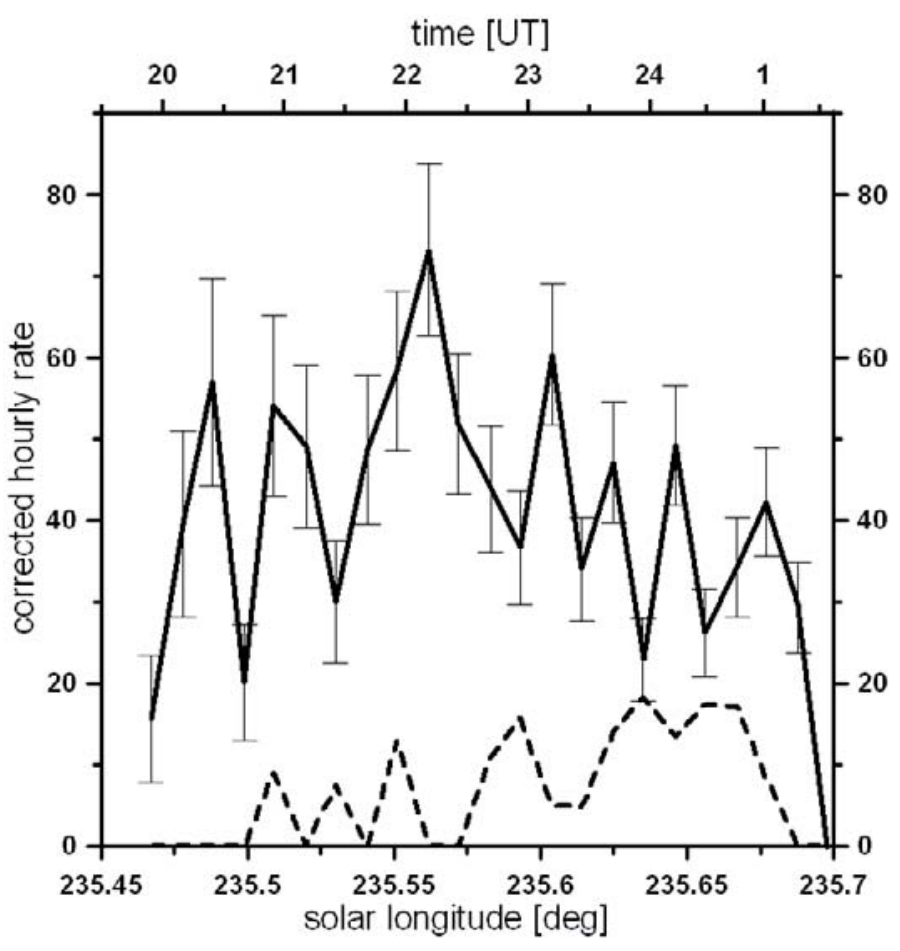

Fig. 2. Corrected instrumental hourly rates of Leonids during both the main night (solid line) and the night before the maximum (dashed line). The annual meteor shower reaches its highest activity more than $12 \mathrm{~h}$ earlier than the main night's peak and did not significantly influence the observed rate during the peak of the observed filament.

(Ceplecha 1998, Sect. 6.2.1). The population index is $r=2.3$. This quantity is useful for determining the flux of meteoroids (see Sect. 3.3).

\subsection{Activity profile}

To be able to say whether the prediction of the modellers was correct, we need to construct the activity curve of the meteor shower. It is usually determined as the time evolution of the number of meteors belonging to the meteor shower. Therefore we used all the available meteors. They were binned into equal time intervals according to the time of occurrence. We tested different lengths of the intervals. The best results were obtained for 15-min long intervals. Shorter intervals were not useful due to the relatively lower number of Leonid meteors, which resulted in big fluctuations of the curve. The correction on the zenith distance $(1 / \cos (z))$ of the radiant was applied on each count of the meteors. The statistical errors were calculated, too. To avoid confusion with the zenith's hourly rate usually determined from the visual observation, we use here the term corrected instrumental hourly rate.

The activity curve is shown in Fig. 2. For illustration the figure also provides the activity of the Leonid meteors during the previous night (from 16 to 17 November) as observed using the video camera at the Gissar observatory. These counts are only from one camera; therefore both curves should not be directly compared. The activity curve shows certain fluctuations resulting from a relatively low total number of the Leonid meteors.

Using different time intervals $(10,20$ or $30 \mathrm{~min})$ did not change results significantly. The maximum of the activity occurred slightly after 22 UT $(22: 07 \pm 00: 15$ UT). Corresponding solar longitude is $\lambda_{O}=235.560^{\circ} \pm 0.010^{\circ}(\mathrm{J} 2000.0)$. The corrected instrumental hourly rate reached $73 \pm 15$ Leonid meteors at the maximum. The shower activity did not, however, show dramatic changes during the full six hours of observations.

\subsection{Flux of the meteoroids}

The meteor rate calculated above is a unique measure for each observational system, because it strongly depends on the meteor limiting magnitude and field of view. We therefore recalculated the activity curve into the flux of the meteoroids brighter than absolute magnitude +6.5 to receive some kind of comparable measure with other observational projects (Brown et al. 2002b).

For this it is necessary to know the populations index (already computed in 3.1) and the area from which all the meteoroids are collected by the camera. Therefore we projected a circular camera's field-of-view with diameter $44^{\circ}$ at the altitude of $106 \mathrm{~km}$. This is the average height of the maximum brightness point of all recorded Leonid meteors. The elevation angle of the camera was $50^{\circ}$. If neglecting the curvature of the Earth, the collecting area has a shape of the ellipse with area of $11500 \mathrm{~km}^{2}$. Because these parameters are unique for each camera we used only meteors observed at one of the stations. Thus 111 Leonid meteors detected by the Kurgan Tube camera were included in the calculation. For this reason, the flux profile is slightly different from the activity profile as presented in previous section.

The flux of meteoroids up to $+6.5^{m}$ can be determined using the equation:

$\Phi_{+6.5}=\Phi_{\mathrm{MLM}} * 10^{(6.5-\mathrm{MLM}) \log r}$,

where MLM is the camera's meteor limiting magnitude, $r$ the population index, and $\Phi_{+6.5}, \Phi_{\mathrm{MLM}}$ is the flux of meteoroids brighter than magnitude +6.5 and MLM, respectively (Brown et al. 2002b). MLM was determined to be +5.0 . The resulting flux of the meteoroids up to magnitude +6.5 is shown in Fig. 3 . At the time of maximum activity the flux reaches a maximum value of $0.02 \pm 0.004$ meteoroids per $\mathrm{km}^{2}$ per $\mathrm{h}$. It was higher than $50 \%$ of the maximum for the period of about $4 \mathrm{~h}$.

\subsection{Flux comparisons}

With knowledge of the flux profile, we can compare with other observations now. Each profile is actually a cross-section of the meteoroid stream, and it provides us with information about the particle density inside it. It allows the theoreticians to test their models backwards.

Table 2 shows the basic parameters of the flux profile as observed during the activity of different Leonid filaments within the past decade.

Even a first glimpse shows that the flux of the meteoroids did not reach the values from the years 1999-2001. It confirms that in 2009 our planet did not meet the dense part of the stream, which is in good agreement with the prediction. In terms of flux, this year Leonid's return instead resembles year 2006. The maximum flux reaches similar values for both 2006 and 2009. Nevertheless, both encounters were still significantly different as Fig. 3 shows. In 2006 we recorded a very sharp and short peak with a total duration of only $1.5 \mathrm{~h}$ and $F W H M$ shorter than $1 \mathrm{~h}$. On the other hand, the 2009 profile shows continue activity of the meteor shower for longer than $5.5 \mathrm{~h}$ but reaches approximately $2 / 3$ of the maxima in 2006 .

These results are consistent with the age of the observed filament. In 2006 we recorded an encounter with a very young filament, because this material was released from the parent comet 


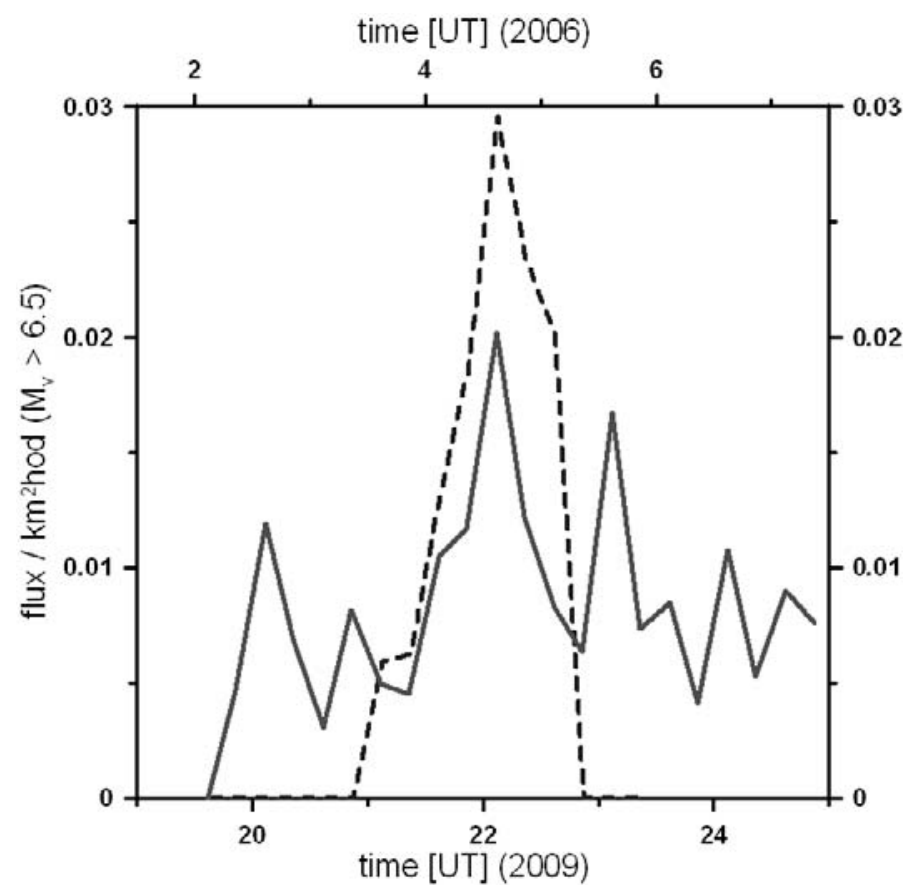

Fig. 3. Flux of the Leonid meteoroids as observed in 2009 from the Kurgan Tube station (solid line) and the comparison with results obtained during 2006 Leonid outburst observed from the Kunzak station in the Czech Republic (dashed line). In both cases the flux is centred on the maximum activity.

Table 2. Comparison of the maximum fluxes for different Leonid meteor stream filaments.

\begin{tabular}{llll}
\hline \hline $\begin{array}{l}\text { Year of } \\
\text { observation }\end{array}$ & Ejection & $\begin{array}{l}\text { Maximum } \Phi_{+6.5}{ }^{* *} \\
{\left[\mathrm{~km}^{-2} \mathrm{~h}^{-1}\right]}\end{array}$ & $\begin{array}{l}\text { Source } \\
\text { of data }\end{array}$ \\
\hline 1999 & 1899 & $0.81 \pm 0.06$ & Brown et al. (2002a) \\
2000 & 1866 & $0.15 \pm 0.02$ & Brown et al. (2002b) \\
& 1733 & $0.11 \pm 0.02$ & Koten (unpublished) \\
2001 & 1767 & 0.35 & $\begin{array}{l}\text { Koten et al. (2008) } \\
2006\end{array}$ \\
2009 & 1932 & $0.03 \pm 0.007$ & this work \\
\hline
\end{tabular}

Notes. ${ }^{(*)}$ Year of the ejection from the parent comet; ${ }^{(*)}$ flux of meteoroids per $\mathrm{km}^{2}$ per $\mathrm{h}$.

only 2 revolutions before the observation, so the stream was still quite compact. Contrasting meteoroids observed in 2009 were ejected more than 500 years ago, and the stream had enough time to become more dispersed. Moreover, the 2006 profile was observed away from the annual Leonid activity, whereas 2009 was projected on it.

\section{Conclusions}

We successfully observed the enhancement of the Leonid meteor shower activity, which was caused by the particles ejected from the parent comet during its perihelion passage in 1466 and 1533. The maximum activity occurred within the predicted time. Although the activity was significantly lower in comparison with big storms that occurred between 1998 and 2002, this detection is still very important. It confirms that modellers are able to predict not only meteor storms but also much smaller events still with very good time precision. Moreover this detection provided us with another profile of the Leonid meteor stream in the vicinity of the Earth orbit.

The activity of the Leonid meteor shower in 2009 has not been reported by any other paper yet. We can only compare with visual data of the International Meteor Organization published on their web page ${ }^{1}$ (IMO). According to this source the peak of activity occurred at 20:19 UT (corresponding solar longitude $\lambda_{O}=235.487^{\circ}$ ) when ZHR reached the value $89 \pm 7$. This is almost two hours earlier than our result. A detailed look at the IMO activity curve shows that the ZHR was high for approximately two hours. The reported IMO peak is represented by only one point, while all the other points show somewhat lower activity. There is also another peak one hour later. Generally the activity was fluctuating between 20 and 22 UT. Finally the results may be influenced by the geographical distribution of the observes. There were only a few of them located the most favoured longitudes (India). Many other observers were positioned too much to the east or west, which would result in significant corrections of the calculated ZHR and strongly influence the results. On the other hand, the activity curve presented in this paper is based on smaller number of meteors.

Another important aspect of observed data is the age of the particles. Observed meteoroids spent a much longer time in interplanetary space than any other Leonid particles, which were observed during the past decade, therefore their physical structure should be influenced more by the spaceweathering and other influences in space. Due to our successful campaign we have very interesting material at our disposal, which will be investigated and compared with other Leonid data originating from younger meteoroids. It will allow us to study how the time of exposure to the spaceweathering could influence the structure of the meteoroids and consecutively their atmospheric behaviour. Results of such a study, including recorded spectra, will be published in another paper.

Acknowledgements. This work was supported by the Grant Agency of the Czech Republic grant No. 205/09/1302. We express our gratitude to Tajik colleagues Mahmud Gulyamov and Umed Hamroev, because without their help our observational campaign should not be carried out. Also we would like to acknowledge the helpful comments of the referee, David Asher.

\section{References}

Brown, P., Campbell, M. D., Hawkes, R. L., Thiejsmeijer, C., \& Jones, J. 2002a, P\&SS, 50, 45

Brown, P., Campbell, M., Suggs, R., et al. 2002b, MNRAS, 335, 473

Ceplecha, Z. 1988, Bull. Astron. Inst. Czechosl., 39, 221

Ceplecha, Z., Borovička, J., Elford, W. G., et al. 1998, Space Sci. Rev., 84, 327

Jenniskens, P., de Kleer, K., Vaubaillon, J., et al. 2008, Icarus, 196, 171

Kondrateva, E. D., \& Reznikov, E. A. 1985, Sol. Sys. Res., 19, 96

Koten, P. 2002, Proc. Asteroids, Comets, Meteors 2002 Conf., ed. B. Warmbein (Berlin), ESA SP, 500, 197

Koten, P., Borovička, J., Spurný, P., et al. 2008, Earth Moon Planets, 102, 151

Lyytinen, E. J., \& Van Flandern, T. 2000, Earth Moon Planets, 82, 149

Maslov M. 2007, WGN, J. Int. Meteor Org., 35, 5

McNaught, R. H., \& Asher, D. J. 1999, WGN, J. Int. Meteor Org., 27, 85

Molau, S. 1999, Proc. Meteoroids 1998 Conf., ed. W. J. Baggaley, \& V. Porubčan (Bratislava), 131

Vaubaillon, J., \& Colas, F. 2005, A\&A, 431, 1139

Vaubaillon, J., Colas, F., \& Jorda, L. 2005, A\&A, 439, 761

\footnotetext{
${ }^{1}$ http://www.imo.net/live/leonids2009/, Meteor Organization, 2009
} 